\title{
Bifurcation Phenomena in Two-Dimensional Piecewise Smooth Discontinuous Maps
}

\author{
Biswambhar Rakshit ${ }^{a}$, Manjul Apratim ${ }^{\text {b }}$, Parag Jain ${ }^{\text {c }}$, \\ Soumitro Banerjee ${ }^{\mathrm{d}, *}$ \\ ${ }^{a}$ Department of Mathematics and Centre for Theoretical Studies, Indian Institute \\ of Technology, Kharagpur-721302, India \\ ${ }^{\mathrm{b}}$ Department of Physics and Astronomy, Rutgers, The State University of New \\ Jersey, NJ 08854-8019 USA \\ c Department of Mechanical Engineering, Stanford University, CA 94305, USA \\ ${ }^{\mathrm{d}}$ Department of Electrical Engineering, Indian Institute of Technology, \\ Kharagpur-721302, India
}

\begin{abstract}
In recent years the theory of border collision bifurcations has been developed for piecewise smooth maps that are continuous across the border, and has been successfully applied to explain nonsmooth bifurcation phenomena in physical systems. However, many switching dynamical systems have been found to yield two-dimensional piecewise smooth maps that are discontinuous across the border. The theory for understanding the bifurcation phenomena in such systems is not available yet. In this paper we present the first approach to the problem of analysing and classifying the bifurcation phenomena in two-dimensional discontinuous maps, based on a piecewise linear approximation in the neighborhood of the border. We explain the bifurcations occurring in a physical system - the static VAR compensator used in electrical power systems, using the theory developed in this paper. This theory may be applied similarly to other systems that yield 2-D discontinuous maps.
\end{abstract}

\section{Introduction}

In recent years the bifurcations occurring in switching dynamical systems have been the subject of great interest, as it is known that many physical, engineering and biological systems actually embody continuous-time evolution

\footnotetext{
* Corresponding author.

Email address: soumitro@ee.iitkgp.ernet.in (Soumitro Banerjee).
} 
punctuated by discrete switching events. Under stroboscopic sampling these switching dynamical systems in general yield maps that have a discontinuity in the derivative along subspaces or "borderlines" that divide the phase space into two or more compartments. In these piecewise smooth (PWS) maps a new type of bifurcation, called border collision bifurcation (1; 2; 3; 4) occurs when a fixed point collides with a borderline, resulting in a sudden change in the Jacobian matrix. Most recent studies on border collision bifurcations have been done on piecewise smooth maps that are continuous across the borderline (2; 5; 6; 7).

It has been reported that many switching dynamical systems yield maps that not only have a discontinuity in the derivative, but also a discontinuity in the function itself. Discontinuity in the map arises if, in the $n$ dimensional Poincaré section there exists an $(n-1)$ dimensional manifold such that infinitesimally close points at the two sides of the manifold map to points that are far apart. A few electronic circuits exhibiting one-dimensional discontinuous maps was given in (8). The bifurcation theory for such one-dimensional discontinuous maps have been developed (9; 10), and have been successfully applied in analyzing bifurcations in physical systems (11). However, in recent years there has been evidence that many physical systems yield two-dimensional (2D) discontinuous maps. For example, the classical impact oscillator yields a $2 \mathrm{D}$ discontinuous map if the impacting surface moves following a nonsmooth function (12). The static VAR controller used in electrical power systems (13; 14) also has its dynamics given by a $2 \mathrm{D}$ discontinuous map. It has also been found that the dynamics of spiking bursting activities of real biological neurons (15; 16) as well as some business cycle models (17) can be represented by 2D piecewise smooth discontinuous maps. Therefore, to explain the bifurcation phenomena in such systems, it is necessary to have a bifurcation theory for $2 \mathrm{D}$ piecewise smooth discontinuous maps.

The bifurcation theory for $1 \mathrm{D}$ and $2 \mathrm{D}$ continuous piecewise smooth maps is well developed (18; 7). The results related to the existence of period-1 and period-2 orbits in general $n$-dimensional PWS maps (5) have also been applied in practical systems. Bifurcation theory for 1D discontinuous maps has been reported (9). In the context of general $n$-dimensional discontinuous maps, some important results related to the existence of period-1 orbits (10) and period-2 orbits (19) have been published recently. For the special case of $n=2$, these theories can be applied to obtain the conditions of existence of period-1 and period-2 fixed points. However in the context of a specific dynamical system, one is often interested in the asymptotically stable behavior, which cannot be inferred from the available theory. It has to be obtained through the analysis of the existence and stability of periodic orbits and their stable and unstable manifolds. Our investigations along this line showed that the two-dimensional discontinuous map is a source of incredibly rich dynamical behavior, created by complicated interactions between the stable and unstable manifolds of fixed 
points and the line of discontinuity. In this paper we report some results of this investigation, and we hope that these will pave way for further investigation on the dynamics of two-dimensional discontinuous maps.

The paper is organized as follows. In Section 2 we present an example of a physical system that yields a discontinuous map, and illustrate the peculiar dynamics exhibited by this system. As in the study of continuous piecewise smooth systems, we use the piecewise linear normal form representing the behavior of the system in the neighborhood of the border-crossing fixed point, which is introduced in Section 3. Then in Section 4 we develop a classification of the bifurcation scenarios observed in this map, depending on the type of the fixed points at the two sides of the border.

\section{A Physical Example: The Reactive Power Compensator}

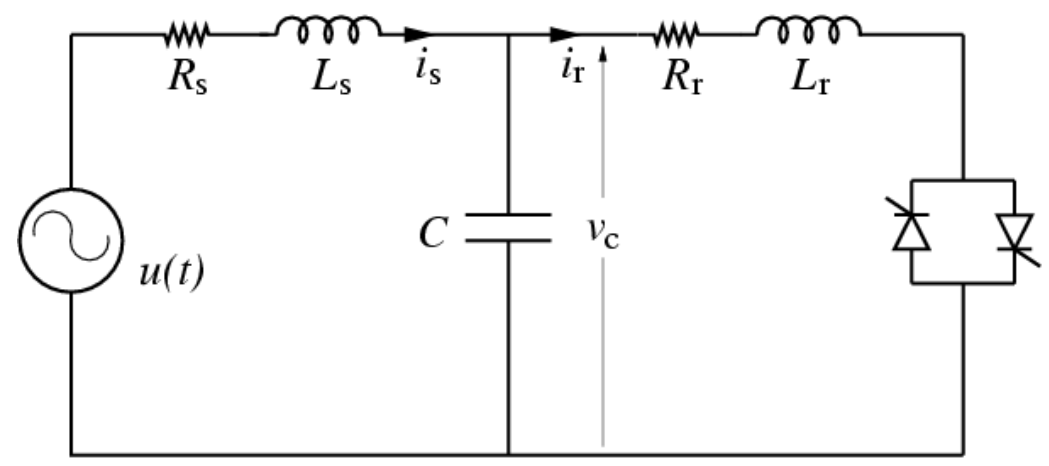

Fig. 1. The Static Var Compensator Circuit. The parameters are $\omega=2 \pi 60 \mathrm{rad} / \mathrm{s}$, $L_{S}=0.195 \mathrm{mH}, R_{s}=0.9 \mathrm{~m} \Omega, L_{r}=1.66 \mathrm{mH}, R_{r}=31.3 \mathrm{~m} \Omega$ and $C=1.5 \mathrm{mF}$.

The system that we shall consider is the static VAR compensator used in electrical power systems, shown in Fig 1. It is an inductor-capacitor combination $\left(L_{r}\right.$ and $C$ ) in which the reactive power consumed by the inductor is controlled by back-to-back connected thyristor switches. The system is connected to a sinusoidal source $u(t)$ at the input (representing the rest of the power system) through a transmission line of inductance $L_{s}$ and resistance $R_{s}$. The two switches operate in the alternate half-cycles, and are turned on by applying pulses at a phase angle $\alpha$ with respect to the sinusoidal input $u(t)=\sin (\omega t)$. They turn off when the current through the respective switches reach a zero value. The system is described by the state vector $\mathbf{x}(t)$ of the current $i_{r}(t)$, capacitor voltage $v_{c}(t)$ and the source current $i_{s}(t)$. The dynamical equation during the on state of either switch is

$$
\dot{\mathbf{x}}=\mathbf{A}_{\mathrm{ON}} \mathbf{x}+\mathbf{B}_{\mathrm{ON}} u
$$




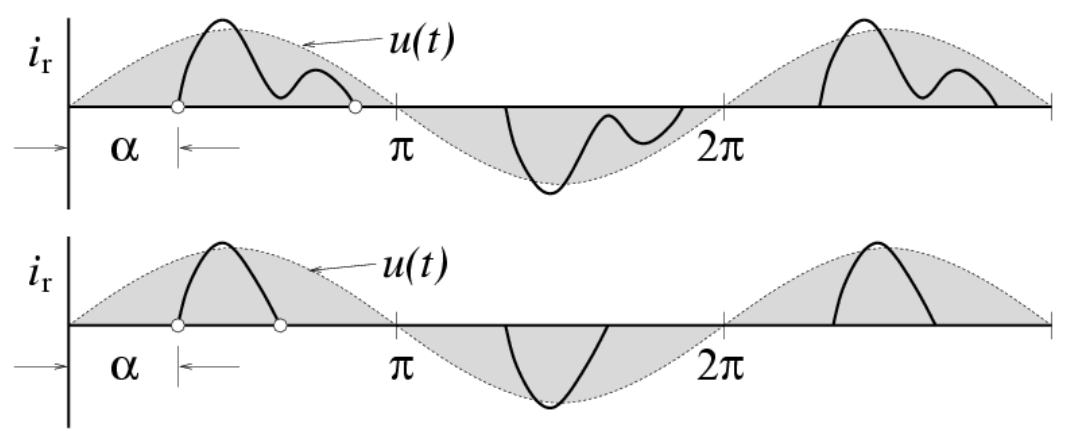

Fig. 2. The $i_{r}$ waveforms for two different initial conditions in $v_{c}$ and $i_{s}$. where:

$$
\mathbf{A}_{\mathrm{ON}}=\left(\begin{array}{ccc}
-R_{r} / L_{r} & 1 / L_{r} & 0 \\
-1 / C & 0 & 1 / C \\
0 & -1 / L_{s} & -R_{s} / L_{s}
\end{array}\right), \quad \mathbf{B}_{\mathrm{ON}}=\left(\begin{array}{c}
0 \\
0 \\
1 / L_{s}
\end{array}\right)
$$

During the OFF phase, $i_{r}(t)$ is zero, and so the state vector is two dimensional, given by $\mathbf{y}=\left[\begin{array}{ll}v_{c} & i_{s}\end{array}\right]^{T}$. The corresponding dynamical equations are

$$
\dot{\mathbf{y}}=\mathbf{A}_{\mathrm{OFF}} \mathbf{y}+\mathbf{B}_{\mathrm{OFF}} u
$$

where

$$
\mathbf{A}_{\mathrm{OFF}}=\left(\begin{array}{cc}
0 & 1 / C \\
-1 / L_{s} & -R_{s} / L_{s}
\end{array}\right), \quad \mathbf{B}_{\mathrm{OFF}}=\left(\begin{array}{c}
0 \\
1 / L_{s}
\end{array}\right)
$$

We obtain the discrete map by observing the state vector stroboscopically, at each positive zero crossing of the input sinusoid. Since the stroboscopic observations are made when the switches are off, the discrete model must be two-dimensional. In this system, the switching logic imposes a discontinuity in the Poincaré map. To illustrate, consider the waveforms of the inductor current $i_{r}$ in Fig. 2. Suppose the switch is turned on at a phase angle $\alpha$ and the waveform of the current $i_{r}$ is as shown. At a slightly different initial condition, the point of dip may reach a zero value, and so the instant of switch-off discontinuously changes as shown in the figure. If for a specific initial condition of $v_{c}$ and $i_{s}$ the waveform just grazes the $x$-axis, then initial conditions at the two sides of this critical value map to widely separated points in the phase space. This implies that the resulting map is discontinuous. For a detailed derivation of the map, refer to $(13 ;$; 14$)$.

Let us now examine the effect of this discontinuity on the system's bifurcation behavior. A typical bifurcation diagram with $\alpha$ as the variable parameter is presented in Fig. 3(a). It is seen that for $\alpha>90^{\circ}$ and for $\alpha<130^{\circ}$, the behavior is period-1. But many peculiar dynamical transitions occur in the intervening parameter range. In such a system it is possible to locate the fixed point, irrespective of its stability, using a shooting method (20). When 

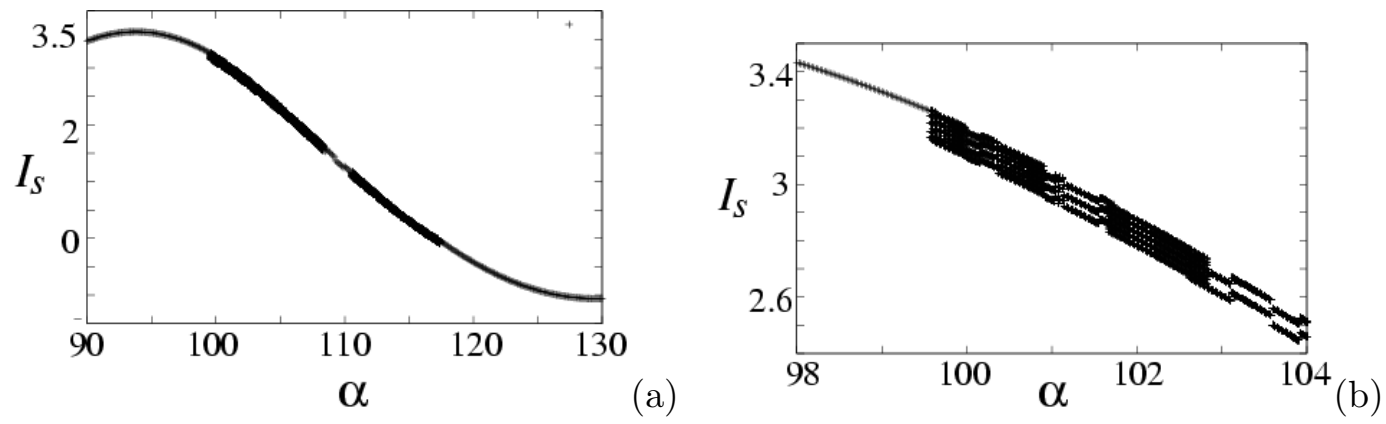

Fig. 3. (a) The bifurcation diagram of the static VAR compensator system. (b) Zoomed portion of the bifurcation diagram in the range $\left(\alpha \in\left[98^{0}, 105^{0}\right]\right)$ showing the occurrence of high-periodic orbits.

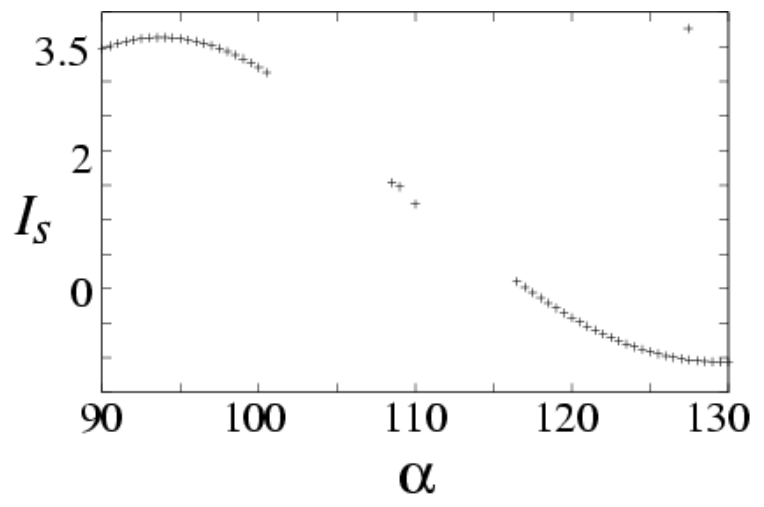

Fig. 4. The bifurcation diagram obtained by following the period-1 orbit using the shooting method.

the bifurcation diagram is drawn by following the periodic orbit (Fig. 4), it is found that there is no fixed point in those parameter ranges. Even though there is no fixed point, the orbit remains bounded and high periodic orbits occur in a specific order (see the zoomed portion in Fig. 3(b)). The objective of this paper is to develop the theory necessary to explain such atypical bifurcation behavior in physical systems.

\section{The Normal Form}

Many hybrid dynamical systems can be represented in discrete-time by piecewise smooth maps, given by equations of the form

$$
f(x, y, \mu)= \begin{cases}g(x, y, \mu), & (x, y) \in R_{A} \\ h(x, y, \mu), & (x, y) \in R_{B}\end{cases}
$$

where $\mu$ is the bifurcation parameter and $R_{A}$ and $R_{B}$ are regions in the state space, divided by a borderline. We consider the class of maps that have the 
following properties:

(1) The functions $g$ and $h$ are smooth (everywhere differentiable),

(2) The function $f$ is discontinuous across the borderline,

(3) The elements of the Jacobian matrix of $f$ change discretely across the borderline,

(4) The Jacobian elements are finite.

Maps of the above properties have application in many physical and engineering systems (the system described in Section 2 provides an example), and in the present paper we restrict our attention to such maps.

The normal form for such a piecewise smooth system in the neighborhood of a fixed point on the border can be expressed as

$$
G(x, y ; \mu)= \begin{cases}\left(\begin{array}{rr}
\tau_{L} & 1 \\
-\delta_{L} & 0
\end{array}\right)\left(\begin{array}{l}
x \\
y
\end{array}\right)+\mu\left(\begin{array}{l}
1 \\
0
\end{array}\right), & x \leq 0 \\
\left(\begin{array}{rr}
\tau_{R} & 1 \\
-\delta_{R} & 0
\end{array}\right)\left(\begin{array}{l}
x \\
y
\end{array}\right)+(\mu+l)\left(\begin{array}{l}
1 \\
0
\end{array}\right), & x>0\end{cases}
$$

where $\tau_{L}, \tau_{R}$ are the traces and $\delta_{L}, \delta_{R}$ are the determinants of the Jacobian matrices at the two sides of the border. The similar normal form of the piecewise smooth continuous map was derived in (1; 6). We have added the discontinuity $l$ to obtain the normal form for the $2 \mathrm{D}$ discontinuous map. We shall call the two halves of the state space $\{x<0\}$ and $\{x>0\}$ as $L$ and $R$ respectively.

In order to explain the bifurcations in a specific system, one has to obtain the eigenvalues of the fixed points at the two sides of the borderline, and from that, the values of the trace and the determinant. For example, in the case of the static VAR compensation system described in Section 2, the calculation of the Floquet exponents at $\alpha=100^{\circ}$ yielded $\tau_{L}=1.4677, \delta_{L}=0.6550$, $\tau_{R}=1.4677$, and $\delta_{R}=0.6549$. The explanation of the observed bifurcations have to be obtained by analyzing the behavior of the normal form map for these parameter values.

The fixed points of the system $(6)$ in both sides of the boundary are given by:

$$
\begin{aligned}
L^{*} & =\left(\frac{\mu}{1+\delta_{L}-\tau_{L}}, \frac{-\delta_{L} \mu}{1+\delta_{L}-\tau_{L}}\right) \\
R^{*} & =\left(\frac{\mu+l}{1+\delta_{R}-\tau_{R}}, \frac{-\delta_{R}(\mu+l)}{1+\delta_{R}-\tau_{R}}\right)
\end{aligned}
$$


If the $x$-component of $L^{*}$ is negative, the fixed point exists. Else it does not. However, when the $x$-component of $L^{*}$ is positive, iterations from initial conditions in the left half are influenced by the "nonexistent" fixed point, which is called a "virtual" fixed point, and is denoted by $\bar{L}^{*}$. Similarly, when the $x$-component of $R^{*}$ is positive, the fixed point exists; else it is a virtual fixed point denoted by $\bar{R}^{*}$.

The stability of $L^{*}$ and $R^{*}$ are determined by the eigenvalues

$$
\lambda_{L \pm}=\frac{1}{2}\left(\tau_{L} \pm \sqrt{\tau_{L}^{2}-4 \delta_{L}}\right), \quad \lambda_{R \pm}=\frac{1}{2}\left(\tau_{R} \pm \sqrt{\tau_{R}^{2}-4 \delta_{R}}\right) .
$$

We shall confine our studies to dissipative systems, so that $\left|\delta_{L}\right|<1$ and $\left|\delta_{R}\right|<1$. Moreover, we shall be concentrating on positive determinant systems because most physical systems are observed to yield maps with positive determinant. For such a map, there can be four basic types of fixed points:

(1) When $-2 \sqrt{\delta}<\tau<2 \sqrt{\delta}$, both eigenvalues of the Jacobian are complex, with moduli less than 1, indicating that the fixed point is a spiral attractor. If $\tau>0$ (real part positive), it is a clockwise spiral, and if $\tau<0$ (real part negative), the motion is counter-clockwise.

(2) When $\delta<\tau^{2} / 4$ and $-(1+\delta)<\tau<(1+\delta)$, both eigenvalues are real and are less than 1 in magnitude, causing the fixed point to be an attractor. If $-(1+\delta)<\tau<-2 \sqrt{\delta}$, then we have $-1<\lambda_{ \pm}<0$, so that the attractor is a flip attractor (flips in both directions). If $2 \sqrt{\delta}<\tau<(1+\delta)$, then $0<\lambda_{+,-}<1$ and the fixed point is a regular attractor.

(3) If $\tau<-(1+\delta)$, then $-1<\lambda_{+}<0$ and $\lambda_{-}<-1$, so that the fixed point is a flip saddle (flips in both direction).

(4) If $\tau>(1+\delta)$, then $\lambda_{+}>1$ and $0<\lambda_{-}<1$, so that the fixed point is a regular saddle.

Earlier work of PWS maps (6) demonstrated a property of the normal form map that the unstable manifolds fold at every intersection with the $x$-axis, and the image of every fold point is a fold point. The stable manifolds fold at every intersection with the $y$-axis and the pre-image of every fold point is a fold point (6, 7). In case of the discontinuous map, the same property holds; the only difference is that the folds are also discontinuous.

\section{Classification of discontinuous bifurcations}

We now classify the different types of discontinuous border collision bifurcations depending on the type of fixed points occurring at the two sides of the border. Unless otherwise stated, we shall study the bifurcations occurring in 
the system (6) as the parameter $\mu$ is varied. Note that while in a continuous map $(l=0)$ border collision occurs at a single value of $\mu$, in the discontinuous case $(l \neq 0)$ border collision occurs at two values $\mu=-l$ and $\mu=0$. Note also that the structure of the map is different for positive and negative values of $l$. So in the subsequent sections we shall consider the bifurcations for positive and negative values of $l$ separately.

If a certain parameter combination exhibits a certain kind of bifurcation behavior upon varying $\mu$ in one direction, then interchanging the parameter values of the $L$ and $R$ sides will yield the same bifurcation behavior upon varying $\mu$ in the reverse direction, i.e., interchanging parameter values is qualitatively mirror-symmetric with respect to the bifurcation behavior.

We now take up each case in the following subsections.

\subsection{Regular Saddle to Regular Saddle \\ $\left(\tau_{L}>1+\delta_{L}, \quad \tau_{R}>1+\delta_{R}\right)$}

Since both the fixed points are unstable, in most situations the orbits starting from all initial conditions go to infinity. However, when the discontinuity is negative, both $L^{*}$ and $R^{*}$ exist in the range $\mu \in(0,-l)$. In such a situation, a trapping region can occur.
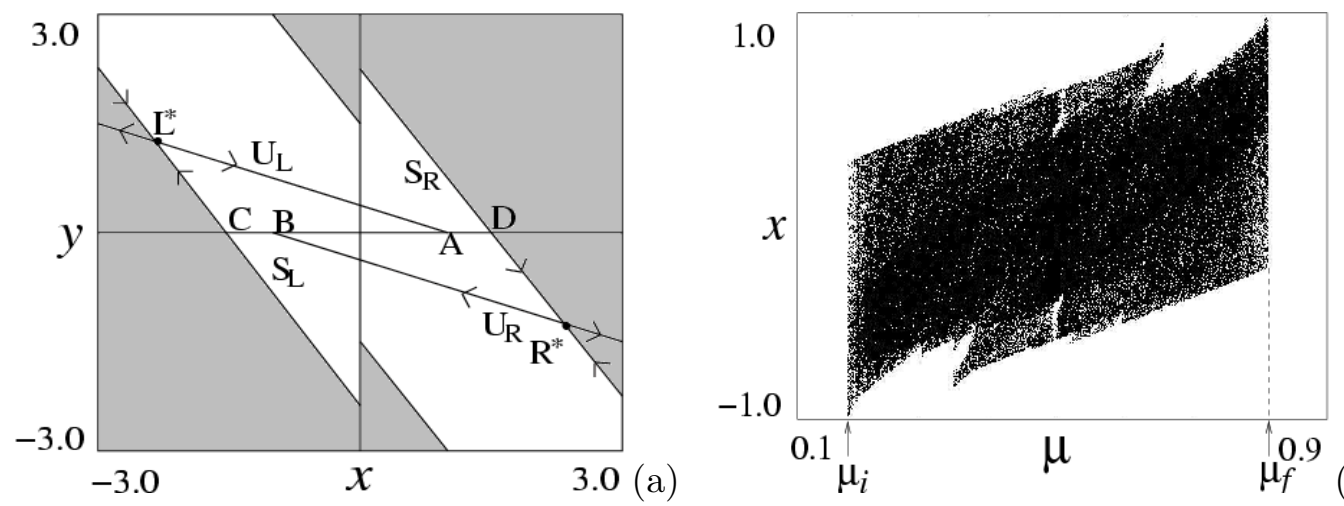

Fig. 5. (a) Structure of the stable and unstable manifolds in the range $\mu \in(0,-l)$ when $L^{*}$ and $R^{*}$ are regular saddles. The parameter values are $\tau_{L}=\tau_{R}=1.5$, $\delta_{L}=\delta_{R}=0.3, l=-1, \mu=0.5$. The trapping region is shown in white. (b) Bifurcation Diagram showing the existence of only a chaotic orbit in the trapping region.

Let $U_{L}$ and $S_{L}$ be the unstable and stable manifolds of $L^{*}$ and $U_{R}$ and $S_{R}$ be the unstable and stable manifolds of $R^{*}$, respectively. The discontinuously folded structure of the manifolds can be seen in Fig. 5(a). Any initial state in $L$ to the right of $S_{L}$ diverges away from $L^{*}$ along $U_{L}$ until it is mapped onto $R$. The state now comes under the influence of $R^{*}$ and is repelled back 
along $U_{R}$. The state therefore gets locked between the two saddles, and this results in a bounded orbit. A similar behavior is seen for an initial state in $R$ to the left of $S_{R}$. Since both sides are stretching, we observe a chaotic orbit. The chaotic attractor is stable as long as the unstable manifolds do not map to states outside the basin of attraction formed by $S_{L}$ and $S_{R}$. This happens in the parameter range $\left[\mu_{i}, \mu_{f}\right]$ where

$$
\mu_{i}=\frac{\frac{-l\left(\lambda_{U R}-1\right)}{1-\tau_{R}+\delta_{R}}}{\frac{1-\lambda_{S L}}{1-\tau_{L}+\delta_{L}}+\frac{\lambda_{U R}-1}{1-\tau_{R}+\delta_{R}}}, \quad \mu_{f}=\frac{\frac{-l\left(1-\lambda_{S R}\right)}{1-\tau_{R}+\delta_{R}}}{\frac{1-\lambda_{S R}}{1-\tau_{R}+\delta_{R}}+\frac{\lambda_{U L}-1}{1-\tau_{L}+\delta_{L}}}
$$

$\lambda_{U R}$ is the eigenvalue of $R^{*}$ outside the unit circle, and $\lambda_{S R}$ is the eigenvalue of $R^{*}$ inside the unit circle. $\lambda_{U L}$ and $\lambda_{S L}$ are the corresponding eigenvalues of $L^{*}$. We will follow the same notations in the subsequent sections. Thus $\mu_{i}$ and $\mu_{f}$ are the parameter values at which boundary crisis occurs, i.e., the unstable manifolds $U_{R}$ and $U_{L}$ touch the stable manifolds $S_{L}$ and $S_{R}$ respectively. We see in Fig. 5(a) that the unstable manifolds $U_{L}$ and $U_{R}$ meet the $x$-axis at $A$ and $B$ respectively while the stable manifolds $S_{L}$ and $S_{R}$ intersect it at $C$ and $D$ respectively. Therefore $\mu_{i}$ is the parameter value after which $B$ lies towards the right of $C$ and $\mu_{f}$ is the value before which $A$ lies towards the left of $D$, rendering the chaotic attractor stable. This range of occurrence of the chaotic attractor is seen in the bifurcation diagram of Fig. 5(b). Note that if the map is continuous, i.e., if $l=0$, the range of occurrence of the chaotic orbit goes to zero, satisfying the situation described in (6).

\subsection{Attractor (Regular/Flip/Spiral) to Regular Saddle$$
\left(-\left(1+\delta_{L}\right)<\tau_{L}<\left(1+\delta_{L}\right), \quad \tau_{R}>1+\delta_{R}\right)
$$

The case of negative discontinuity: For $\mu<0$, both $L^{*}$ and $R^{*}$ are real and we have a period-1 orbit. $L^{*}$ can be a regular/flip/spiral attractor depending on the relationship between $\tau_{L}$ and $\delta_{L}$. The period-1 orbit is unique for $\mu<0$ if $L^{*}$ is a regular attractor. However, high-periodic orbits as well as chaotic orbit may coexist with the period-1 orbit for values of $\mu$ slightly less than 0 if $L^{*}$ is a spiral or flip attractor. When $L^{*}$ is very close to the $y$-axis, any trajectory approaching it has a possibility of going over to $R$ - which happens for spiralling or flipping orbits.

For $\mu \in(0,-l), \bar{L}^{*}$ is a virtual attractor, and $R^{*}$ is a regular saddle. The stable manifold $S_{R}$ of $R^{*}$ is responsible for forming the basin boundary, while the virtual attractor $\bar{L}^{*}$ present in $R$ draws the states in $L$ within the basin of attraction towards $R$ (see Fig. 6(a), Fig. 7(a), Fig. 8(a)). Any initial state in $R$ to the left of $S_{R}$ diverges asymptotically along the unstable manifold $U_{R}$ until it is mapped onto $L$. It now comes back under the influence of $\bar{L}^{*}$ and is drawn back to $R$. The trajectory therefore gets locked between the saddle and 
the virtual attractor resulting in a bounded orbit. This orbit is stable before a parameter value $\mu_{c}$ after which the unstable manifold $U_{R}$ maps to points outside the basin boundary formed by $S_{R}$, i.e., a boundary crisis occurs at $\mu=\mu_{c}$ as $\mu$ is increased. The value of $\mu_{c}$ depends on the slope of the unstable
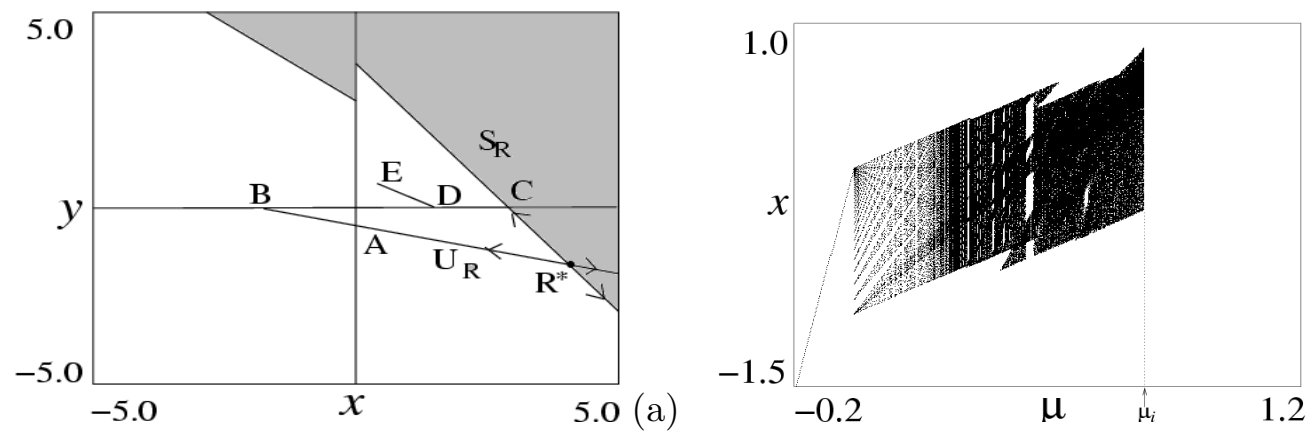

Fig. 6. (a) The structure of stable and unstable manifolds when $m_{U R 1}>m_{S R}$ and it is negative, $\tau_{L}=1.0, \tau_{R}=1.0, \delta_{L}=\delta_{R}=0.3, l=-1, \mu=0.2$, and (b) Bifurcation diagram with $\tau_{L}=1.0, \tau_{R}=1.5, \delta_{L}=0.1, \delta_{R}=0.2, l=-1$.

manifold after it first folds discontinuously at the $x$-axis. This slope after the first fold is $m_{U R 1}=\delta_{L} /\left(\lambda_{S R}-\tau_{L}\right)$ (Here $\left.\lambda_{S R}=\lambda_{R-}\right)$. When $m_{U R 1}>m_{S R}$, since both the slopes are negative, the two manifolds can never intersect (see Fig. 6(a)).

We see that $U_{R}$ intersects the $y$-axis at $A$, and arbitrarily close points at the right and left of $A$ map discontinuously to $B$ and $D$ respectively. The attractor becomes unstable due to boundary crisis when $D$ moves to the right of $C$ on the $x$-axis. This gives $\mu_{c}$ as

$$
\mu_{c}=\mu_{i}=\frac{\left(2 \lambda_{S R}-\delta_{R}-1\right) l}{\left(\lambda_{U R}-\lambda_{S R}\right)}
$$

which is positive. However, under the conditions depicted in Fig. 7)(a) and
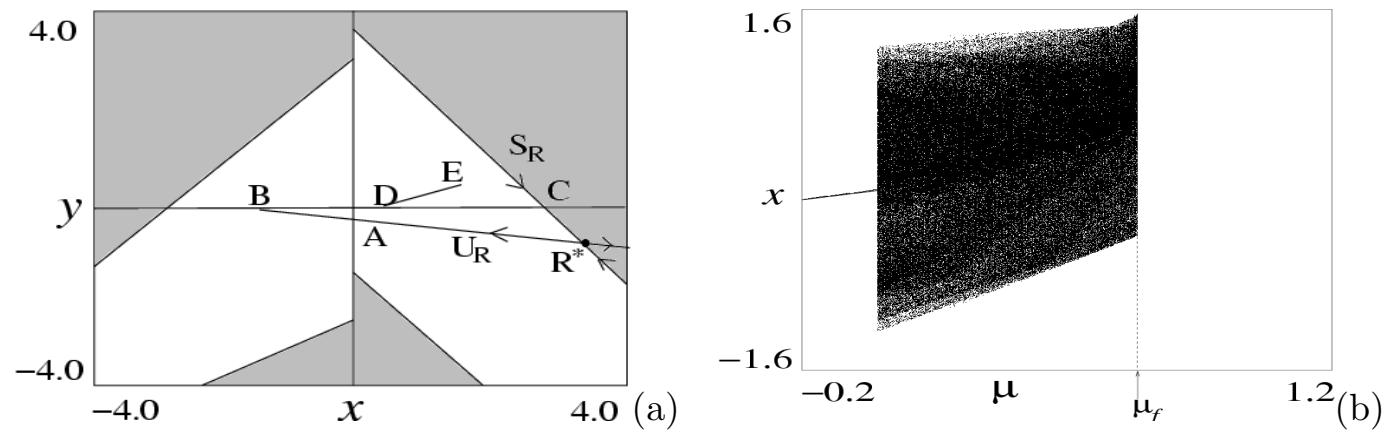

Fig. 7. (a) The structure of stable and unstable manifolds when $m_{U R 1}>m_{S R}$ but it is Positive, $\tau_{L}=-1.0, \tau_{R}=1.0, \delta_{L}=\delta_{R}=0.3, l=-1, \mu=0.2$, and(b) Bifurcation diagram with $\tau_{L}=-1.0, \tau_{R}=1.5, \delta_{L}=\delta_{R}=0.3, l=-1$.

Fig. 8(a), boundary crisis occurs when the segement of $U_{R}$ after the first fold intersects with $S_{R}$. We see from the corresponding Fig. 7)(a) and Fig. 8(a) that 
$U_{R}$ cuts the $x$-axis at $B$, and $B$ maps to $E$. Thus the bounded orbit becomes unstable when $E$ touches $S_{R}$. For this condition, $\mu_{c}$ is given by

$$
\mu_{c}=\mu_{f}=\frac{\left[\lambda_{U R}+\delta_{L}\left(1-\lambda_{U R}\right)+\tau_{L} \lambda_{U R}\left(1-\lambda_{U R}\right)-\lambda_{U R} \tau_{R}\right] l}{\left[\delta_{R}\left(1+\lambda_{U R}\right)-\delta_{L}\left(1-\lambda_{U R}\right)+\tau_{L} \lambda_{U R}\left(1-\lambda_{U R}\right)-\lambda_{U R} \tau_{R}\right]}
$$
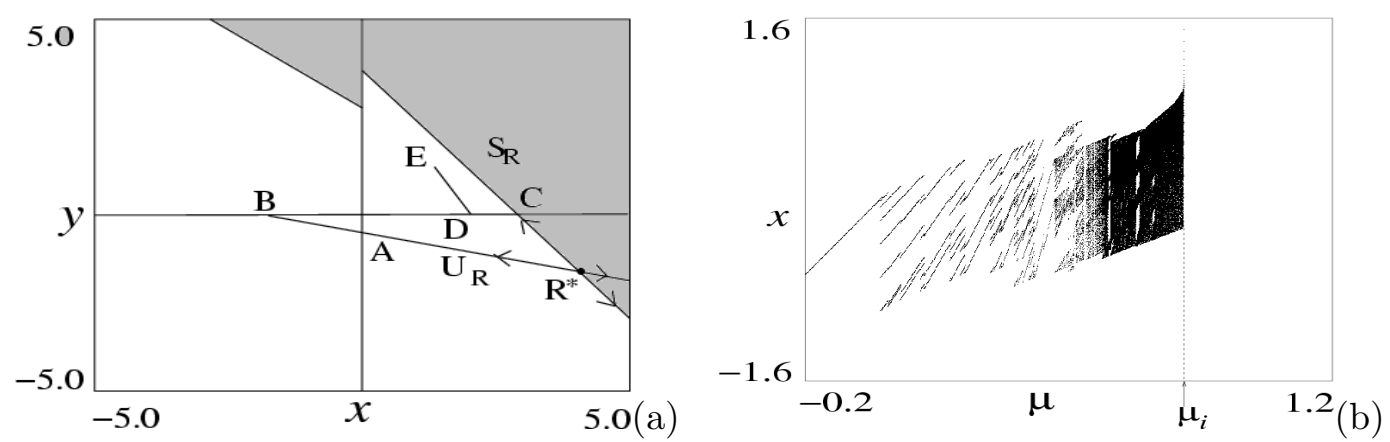

Fig. 8. (a)The structure of stable and unstable manifolds when $m_{U R 1}<m_{S R}$, $\tau_{L}=0.30, \tau_{R}=1.0, \delta_{L}=\delta_{R}=0.3, l=-1, \mu=0.2$, and (b) Bifurcation diagram with $\tau_{L}=0.30, \tau_{R}=1.5, \delta_{L}=\delta_{R}=0.3, l=-1$.

As $\mu$ is increased through zero, the period-1 orbit vanishes through BCB and high periodic orbits of type $L^{n} R$ come into existence (Fig. 6(b) and Fig. 8(b)) with period increment and Farey tree sequence. With further increase of $\mu$ symbol sequence gets reversed, i.e., the symbol sequence of type $L R^{n}$ come into existence with period adding and Farey tree. But since $\tau_{R}>\left(1+\delta_{R}\right)$, eventually the orbit becomes globally stretching, leading to chaotic dynamics at some value of $\mu$. Depending on the value of $\tau_{L}$ a direct transition to chaos is also possible (Fig. $7(\mathrm{~b})$ ). In both the cases chaotic attractor disappears due to a boundary crisis.

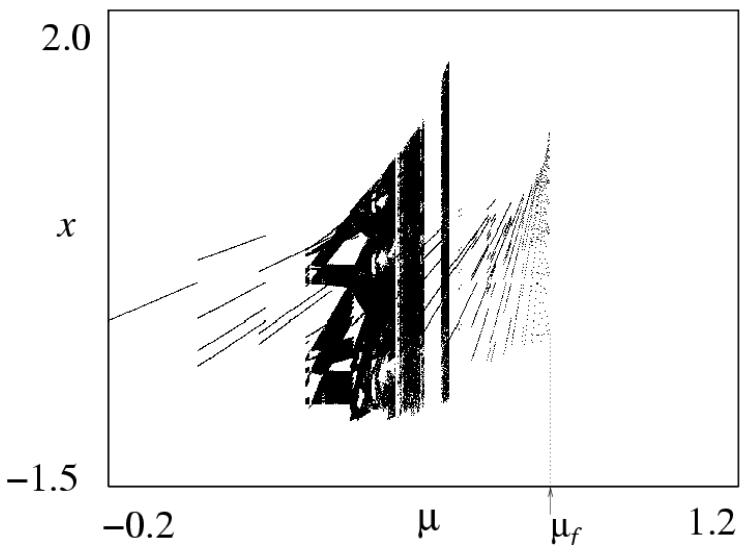

Fig. 9. Bifurcation diagram with $\tau_{L}=1.0, \tau_{R}=1.9, \delta_{L}=\delta_{R}=0.7, l=-1$.

In the $\tau_{L}-\tau_{R}$ parameter space, the domains of occurrence of each periodic orbit depends on the value of the determinants. For higher values of the determinant the regions overlap, resulting in coexistence of attractors and chaotic inclusions (see Fig. 9). 
For $\mu>-l, L^{*}$ and $R^{*}$ are both virtual, which results in the entire state space being unstable because initial conditions in $R$ diverge to infinity under the action of the unstable manifold (directed away from $L$ to infinity) of the nonexistent fixed point $\bar{R}^{*}$, now located inside $L$, and initial conditions in $L$ are also attracted to the side $R$ due to the action of the nonexistent virtual attractor $\bar{L}^{*}$.

For the case of positive discontinuity, we have a period-1 attractor in the case when both $L^{*}$ and $R^{*}$ are real (for $\mu<-l$ ), else the entire space is unstable.

The parameter space is symmetric upon variation of $\mu$ in the reverse direction when $L^{*}$ is a regular saddle and $R^{*}$ is an attractor, i.e., for $\tau_{L}>\left(1+\delta_{L}\right)$ and $-\left(1+\delta_{R}\right)<\tau_{R}<\left(1+\delta_{R}\right)$. In that case a chaotic attractor is initially born out of a boundary crisis, slowly giving rise to increasingly stable orbits of non-monotonically decreasing periodicities, ultimately leading to a stable period-1 orbit.

\subsection{Regular Saddle to Flip Saddle \\ $\left(\tau_{L}>1+\delta_{L}, \quad \tau_{R}<-\left(1+\delta_{R}\right)\right)$}

The stable manifold $S_{L}$ of the regular saddle in $L$ is responsible for forming the boundary of the basin of attraction, while the flip saddle in $R$ is located inside this basin and is responsible for creating the attractor. This leads to many interesting phenomena as we shall see.

The case of negative discontinuity: For $\mu<0$, when both saddles are virtual, we do not observe any attractor. For $\mu>-l, L^{*}$ is a regular saddle and $R^{*}$ is a flip saddle. All initial conditions in $L$ converge onto the unstable manifold $U_{L}$. An initial condition to the right of the basin boundary formed by $S_{L}$ converges onto the unstable manifold $U_{L}$ and is mapped into $R$ (see Fig. 10(a)). The slope of the unstable manifold of $R^{*}$ is $m_{U R}=-\delta_{R} / \lambda_{U R}=-\lambda_{R+}$ while the slope of the stable manifold is $m_{S R}=-\lambda_{R-}$. The unstable manifold $U_{R}$ folds discontinuously at the intersection with the $x$-axis and continues with a slope $m_{U R}=\delta_{L} \lambda_{U_{R}} /\left(\delta_{R}-\tau_{L} \lambda_{U R}\right)=\delta_{L} \lambda_{R-} /\left(\delta_{R}-\tau_{L} \lambda_{R-}\right)$. Since $m_{S R}>$ 0 and $m_{U R}<0$ (as dictated by the range of parameters in question), the unstable manifold $U_{R}$ must have a transverse homoclinic intersection with the stable manifold $S_{R}$ after the first fold, which implies an infinity of homoclinic intersections and the existence of a chaotic orbit. For the regular saddle $L^{*}$, we note that the unstable manifold $U_{L}$ has a negative slope $m_{U L}=-\lambda_{L+}$, and must, therefore, also have a heteroclinic intersection with the stable manifold $S_{R}$ of the flip saddle $R^{*}$. The Lambda Lemma (21) implies that the unstable manifolds of the two sides will come arbitrarily close to each other, which makes the chaotic attractor robust (22). 

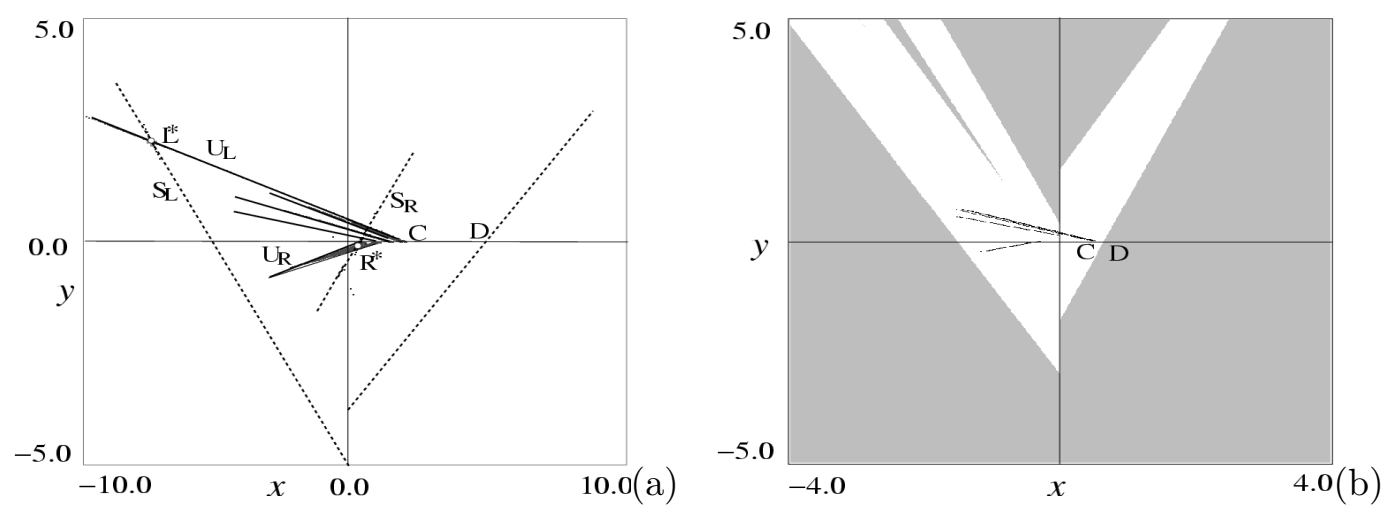

Fig. 10. (a) Homoclinic and heteroclinic intersections of the stable and unstable manifolds of the flip saddle $R^{*}$ when $\tau_{L}=1.5, \tau_{R}=-1.5, \delta_{L}=\delta_{R}=0.3$, $l=-1, \mu=1.5$. This figure and the subsequent ones showing the invariant manifolds have been generated using DsTool (23, 24; 25). (b) The chaotic attractor, and the basin of attraction created by the stable manifold of $L^{*}$, for $\tau_{L}=1.5, \tau_{R}=-1.5, \delta_{L}=\delta_{R}=0.3, l=-1, \mu=0.5$.

It is clear from the geometric structure that no point of the attractor can be to the right of point $C$. If $C$ lies towards the left of $D$, the chaotic orbit is stable. If $C$ falls outside the basin of attraction, it is an unstable chaotic orbit. From this we obtain the critical value of $\mu$ for which boundary crisis will occur:

$$
\mu_{i}=\frac{-\lambda_{U_{L}}\left(1-\tau_{L}+\delta_{L}\right) l}{\lambda_{U_{L}}\left(\lambda_{S_{L}}-\tau_{L}+\delta_{L}\right)+\left(\lambda_{U_{L}}-1\right)\left(\delta_{R}-\lambda_{U_{L}} \tau_{R}\right)}
$$

which is the same as 10 taken in the reverse direction of $\mu$ variation. Note that for a continuous map the occurrence of the boundary crisis does not depend on $\mu$, but in the discontinuous map it does.

The same phenomenon occurs for $0<\mu<-l$ where $\bar{R}^{*}$ is a virtual flip saddle, but its stable and unstable manifolds still exist in $R$ and undergo homoclinic intersections resulting in chaos. The chaotic orbit occurs inside the basin of attraction formed by the stable manifold of $L^{*}$ (see Fig. $10(\mathrm{~b})$ ).

The case of positive discontinuity: For $\mu>0, L^{*}$ is a regular saddle and $R^{*}$ is a flip saddle. In this case the same mechanism as discussed above causes a chaotic attractor, with the same stability condition as in (11).

When there is a transition from a flip saddle to a regular saddle, i.e., when $\tau_{L}<-\left(1+\delta_{L}\right)$ and $\tau_{R}>1+\delta_{R}$, the bifurcation behavior is the same if $\mu$ is varied in the opposite direction. 


\subsection{Attractor to Attractor (Regular/Flip/Spiral) \\ $-\left(1+\delta_{L}\right)<\tau_{L}<\left(1+\delta_{L}\right), \quad-\left(1+\delta_{R}\right)<\tau_{R}<\left(1+\delta_{R}\right)$}

Once again, in this case, we shall be led to observe phenomena that do not occur in smooth maps or piecewise smooth continuous maps.

The case of negative discontinuity: For both $\mu<0$ and $\mu>-l$, when one of the attractors is real and the other virtual, a stable period- 1 orbit exists. If the fixed point is a spiral or flip attractor, high-periodic orbits may coexist with the period- 1 orbit for values of $\mu$ slightly less than zero or slightly greater than $-l$, because initial conditions close to the border may map to the other side before converging on the fixed point.
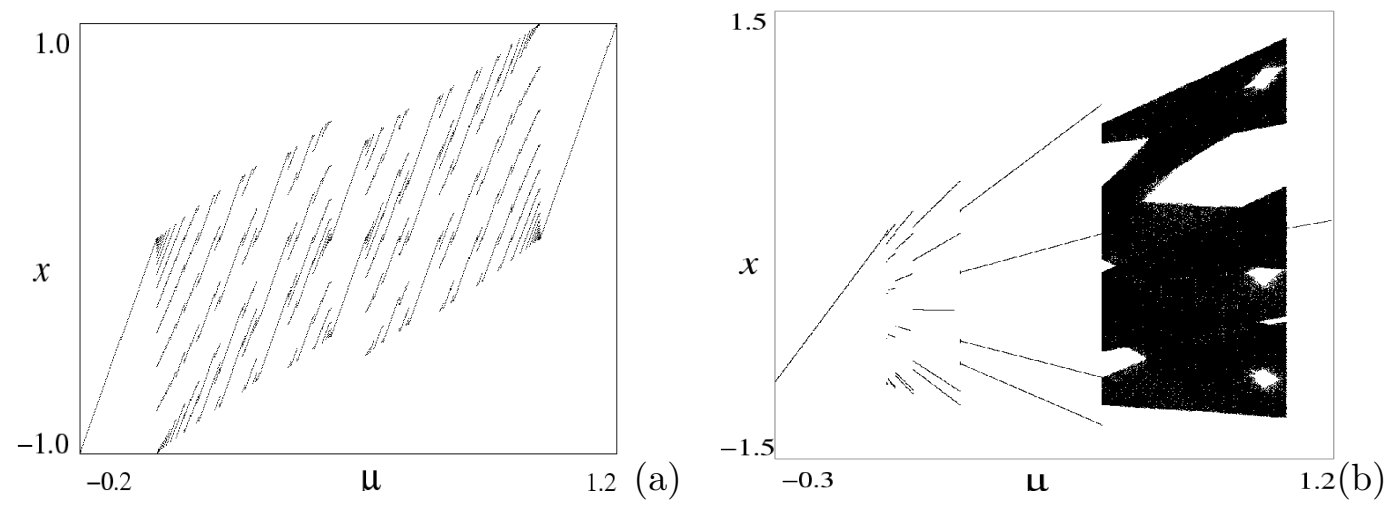

Fig. 11. (a) Bifurcation Diagram when both $\tau_{L}$ and $\tau_{R}$ are positive, with $\tau_{L}=1.0$, $\delta_{L}=0.2, \tau_{R}=1$., $\delta_{R}=0.2$, and $l=-1$. (b) Bifurcation Diagram when $\tau_{L}$ is positive and $\tau_{R}$ is negative, with $\tau_{L}=1.0, \tau_{R}=-1.0, \delta_{L}=\delta_{R}=0.3, l=-1$.

For $\mu \in(0,-l)$, both $\bar{L}^{*}$ and $\bar{R}^{*}$ are virtual attractors. Thus any initial state in $L$ is drawn towards the virtual attractor $\bar{L}^{*}$ situated in $R$. However as soon as it crosses the $y$-axis, it is drawn back towards the virtual attractor $\bar{R}^{*}$ situated in $L$. The state therefore gets locked between the two virtual attractors and that results in a bounded orbit. The same behavior is exhibited by an initial state in $R$. This closed orbit may be high-periodic or chaotic. For $0<\tau_{L}<\left(1+\delta_{L}\right)$ and $0<\tau_{R}<\left(1+\delta_{R}\right)$, the bifurcation diagram in Fig. 11(a) shows that as $\mu$ is increased through zero period-1 orbit vanishes through border collision bifurcation and high periodic orbits of type $L^{n} R$ come into existence with period inclusion and Farey tree sequence. With further increase of $\mu$, the symbol sequence gets reversed, i.e., the symbol sequence of type $L R^{n}$ comes into existence. Finally a period-1 orbit come into existence through BCB.

We have seen that in a discontinuous map a parameter range can exist where both the fixed points are virtual, and if both the virtual fixed points are attracting in nature, high periodic orbits occur in specific sequence.

However for higher values of the determinants $\left(\delta_{L}, \delta_{R}\right)$, the bifurcation struc- 

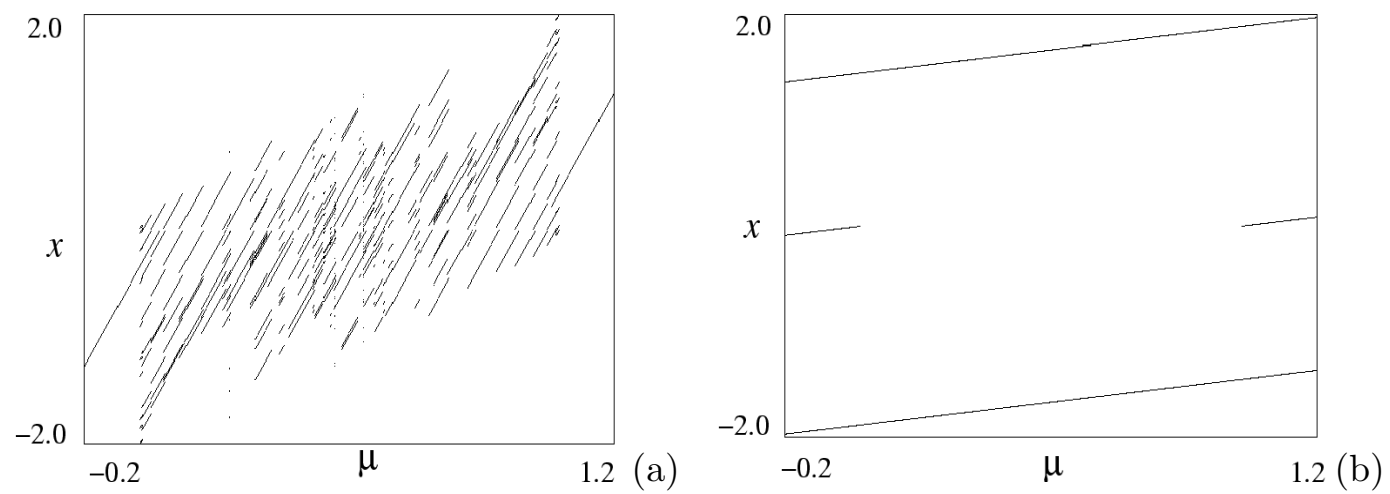

Fig. 12. (a) Bifurcation Diagram when both $\tau_{L}$ and $\tau_{R}$ are positive, with $\tau_{L}=\tau_{R}=1.4677, \delta_{L}=\delta_{R}=0.655, l=-1$. (b) Bifurcation Diagram when both $\tau_{L}$ and $\tau_{R}$ are negative, with $\tau_{L}=\tau_{R}=-1.0, \delta_{L}=\delta_{R}=0.3, l=-1$.

ture is more complex. Here we can observe period increment with coexistence of attractors. Notice that in Fig. 12(a) the parameters are the same as the ones obtained for the physical system considered in Section 2. The traces and determinants obtained from the physical system indicate that the local behavior of the system satisfies the condition being discussed in this section. The dynamical phenomena observed in Fig. 3 and in Fig. 12(a) are qualitatively the same, which explains the peculiar dynamical behaviors observed in the static VAR controller system.
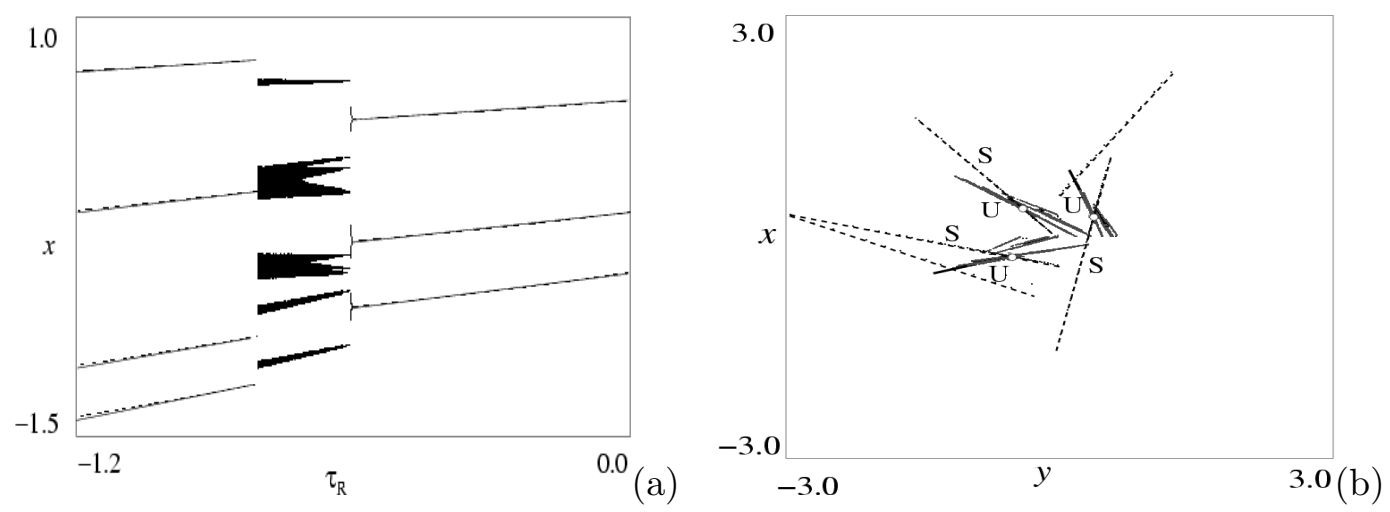

Fig. 13. (a) Bifurcation diagram with respect to $\tau_{R}$, for $\tau_{L}=1.0, \delta_{L}=\delta_{R}=0.3$, $l=-1, \mu=0.5$. (b) Homoclinic intersection of stable and unstable manifolds of a period-3 saddle.

For $0<\tau_{L}<\left(1+\delta_{L}\right)$ and $-\left(1+\delta_{R}\right)<\tau_{R}<0$, the high period orbits do not occur throughout the parameter range and a chaotic orbit is found to occur (see the bifurcation diagram in Fig. 111(b)) for high values of $\mu$. This is a novel phenomenon - the occurrence of chaos in a system where both parts of the state space are contractive. The mechanism of the creation of the chaotic orbit becomes clear from the bifurcation diagram with $\tau_{R}$ as the parameter (see Fig. 13(a)). As the parameter is reduced, the period-3 orbit's eigenvalues reach -1 and it undergoes a period doubling. The resulting period-6 orbit hits the border and turns into a six-piece chaotic orbit. The period-3 fixed point 
is now a saddle, and Fig. 13(b) shows that its stable and unstable manifolds undergo a homoclinic intersection. This gives rise to the chaotic orbit. At a lower value of the parameter, a period-4 orbit starts to exist, initially coexisting with the chaotic orbit. At a specific parameter value, a boundary crisis occurs and the chaotic attractor disappears. At other parameter ranges, a similar phenomenon occurs for the other high-periodic fixed points.

For $-\left(1+\delta_{L}\right)<\tau_{L}<0$ and $-\left(1+\delta_{R}\right)<\tau_{R}<0$, i.e., when both the fixed points are flip attractors, a different situation occurs. In the parameter range $\mu \in(0,-l)$ when no fixed point exists (both are virtual), only a period- 2 orbit occurs (see Fig. 12(b)). In fact, the range of occurrence of the period-2 orbit extends beyond this range, and the period- 2 orbit coexists with the period-1 orbit over a significant parameter range. Note that in the case of a continuous map, a stable period-2 orbit cannot occur when the map is globally contractive (5; 6).

A symmetric behavior is exhibited for $-\left(1+\delta_{R}\right)<\tau_{R}<0$ and $0<\tau_{L}<$ $\left(1+\delta_{L}\right)$ upon varying $\mu$ in the reverse direction.
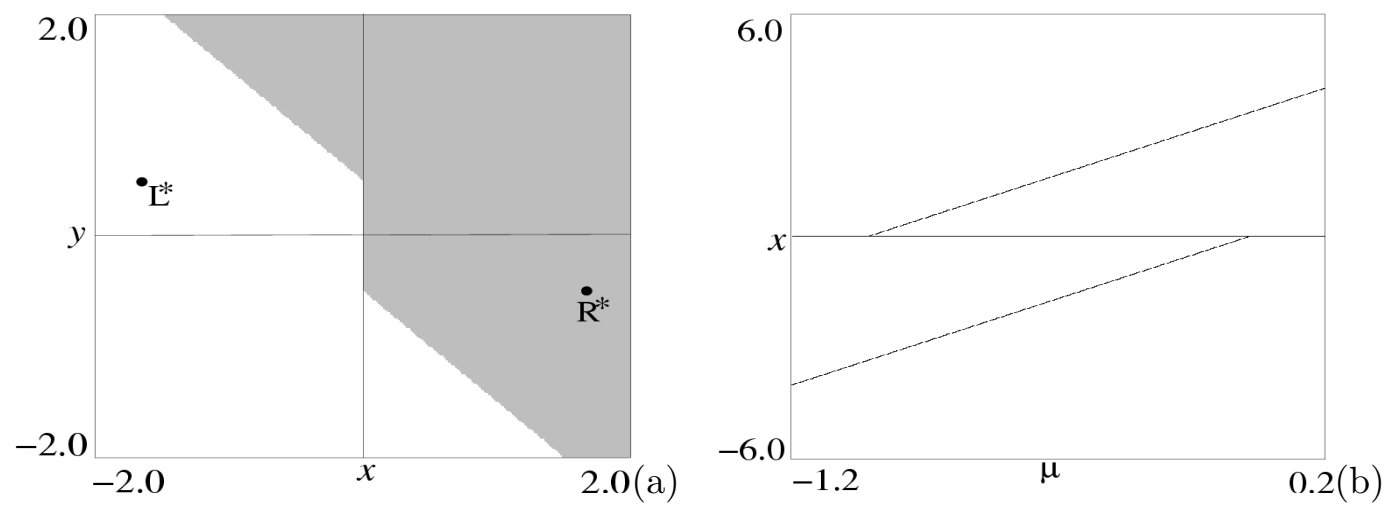

Fig. 14. (a) Basin of attraction, for $\tau_{L}=1.0, \tau_{R}=1.0, \delta_{L}=\delta_{R}=0.3, \mu=0.5$, $l=1.0$. (b) Bifurcation diagram with respect to $\mu$, for $\tau_{L}=1.0, \tau_{R}=1.0$, $\delta_{L}=\delta_{R}=0.3, l=1.0$.

The case of positive discontinuity: In this case there is one real attracting fixed point in each of the parameter ranges $\mu<-l$ and $\mu>0$. For $\mu \in(0,-l)$ both $L^{*}$ and $R^{*}$ are attracting fixed points, and have their own basins of attraction. It is known that the basin boundary is normally formed by the stable manifold of a saddle fixed point. However, under the condition $0<\tau_{L}<\left(1+\delta_{L}\right)$ and $0<\tau_{R}<\left(1+\delta_{R}\right)$ the basin boundary is formed by a completely different mechanism. In this case the segment of the borderline from $-(\mu+l)$ and $-\mu$ (the two preiterates of the origin) form a part of the basin boundary. The rest of the basin boundary is formed by successive preiterates of this segment, as can be seen in Fig. 14(a). This is due to the fact that in a discontinuous map, the borderline can act as a repellor. Moreover, its preiterates can also act as repellors since two points at the two sides of these line segments eventually map to points far apart. This mechanism gives rise to the bifurcation diagram 
of Fig. 14(b).

4.5 Regular Attractor to Flip Saddle

$\left(2 \sqrt{\delta_{L}}<\tau_{L}<\left(1+\delta_{L}\right), \quad \tau_{R}<-\left(1+\delta_{R}\right)\right)$

The Case of Negative Discontinuity: For $\mu<0, L^{*}$ is a regular attractor and $\bar{R}^{*}$ is a virtual flip saddle, and the attracting fixed point enforces a stable period-1 orbit since all initial conditions eventually lead to it.

For $\mu>-l, \bar{L}^{*}$ is a virtual attractor, and hence lies in $R . R^{*}$ on the other hand is a flip saddle, and its stable and unstable manifolds undergo homoclinic intersections after the first fold (see Fig. 15(a)) to yield a chaotic attractor. After the first fold of $U_{R}$, its slope becomes $\delta_{L} \lambda_{U_{R}} /\left(\delta_{R}-\tau_{L} \lambda_{U_{R}}\right)$ (a negative value), while the slope of $S_{R}$ given by $-\lambda_{U_{R}}$ remains positive. All initial conditions in $L$ are drawn to $R$ due to the action of the virtual attractor $\bar{L}^{*}$, where they converge onto the chaotic attractor. This chaotic attractor extends into the parameter range $0<\mu<-l$, when $\bar{R}^{*}$ becomes virtual, but its stable and unstable manifolds still exist in $R$ and undergo homoclinic intersections to yield a chaotic orbit. Hence, any initial condition in the entire phase space ultimately converges on the chaotic attractor. The chaotic attractor manifests for most of the parameter space, but for values of $\mu$ slightly greater than zero some high-periodic orbits are also stable.
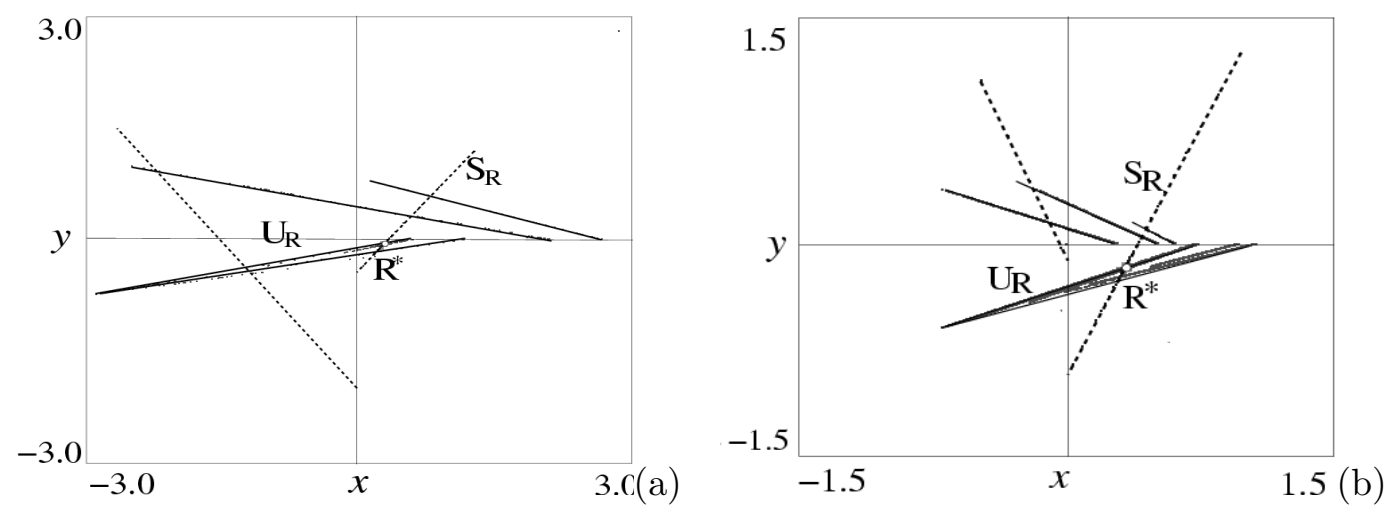

Fig. 15. (a) Homoclinic intersections of stable and unstable manifolds of the flip saddle $R^{*}$ for a negative discontinuity, with $\tau_{L}=1.0, \tau_{R}=-1.0, \delta_{L}=\delta_{R}=0.3, l=-1, \mu=0.5$. (b) Homoclinic intersections of stable and unstable manifolds of the flip saddle $R^{*}$ for a positive discontinuity, with $\tau_{L}=1.2, \tau_{R}=-1.5, \delta_{L}=\delta_{R}=0.3, l=1, \mu=0.5$.

The Case of Positive Discontinuity: For $\mu<-l, L^{*}$ is a regular attractor and $\bar{R}^{*}$ is a virtual flip saddle; for $\mu \in(-l, 0), L^{*}$ is a regular attractor and $R^{*}$ is a flip saddle; for $\mu>0, \bar{L}^{*}$ is a virtual regular attractor and $R^{*}$ is a flip saddle. For the first two cases, the regular attractor in $L$ causes a period-1 orbit to exist for all initial conditions. For the case when $\bar{L}^{*}$ is a virtual attractor and 
$R^{*}$ is a flip saddle, the stable and unstable manifolds of $R^{*}$ undergo homoclinic intersection (Fig. 15(b)) to yield a chaotic orbit.

\subsection{Spiral Attractor to Flip Saddle \\ $\left(-2 \sqrt{\delta_{L}}<\tau_{L}<2 \sqrt{\delta_{L}}, \quad \tau_{R}<-\left(1+\delta_{R}\right)\right)$}

The Case of Negative Discontinuity: For $\mu<0, L^{*}$ is a spiral attractor and $\bar{R}^{*}$ a virtual flip saddle, and the attractor causes a period-1 orbit. Whenever the spiral attractor is very close to the $y$-axis (which happens either when $\mu$ slightly less than zero or $\tau_{L}$ is negative), high-periodic orbits coexist with the period-1 orbit (see Fig. 16(a)).
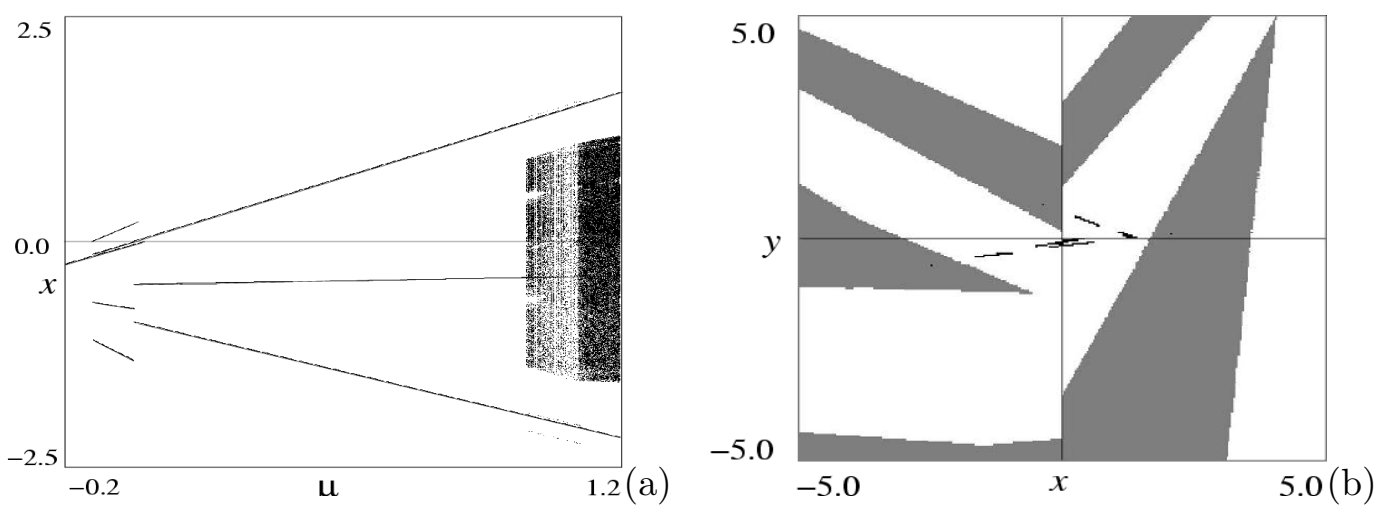

Fig. 16. (a) Bifurcation Diagram with $\tau_{L}=0.5, \tau_{R}=-1.5, \delta_{L}=\delta_{R}=0.3, l=-1$. (b) Basin of Attraction with $\tau_{L}=0.5, \tau_{R}=-1.5, \delta_{L}=\delta_{R}=0.3, l=-1, \mu=1.2$.

High-periodic orbits continue to exist for $\mu \in(0,-l)$ when $\bar{L}^{*}$ is a virtual attractor and $\bar{R}^{*}$ is a virtual flip saddle, because initial conditions in $L$ are mapped to $R$ due to the virtual attractor $\bar{L}^{*}$ in $R$, and points in $R$ are then mapped back to $L$ due to action of the virtual flip saddle $\bar{R}^{*}$, thus leading to a bounded orbit, which manifests as a high-periodic or chaotic orbit (chaos occurs only when $\tau_{L}$ is positive).

For $\mu>-l, \bar{L}^{*}$ is a virtual attractor while $R^{*}$ is a flip saddle. The virtual attractor $\bar{L}^{*}$ maps all initial conditions in $L$ to $R$, which subsequently flip to $L$. This may either lead to a stable high-periodic orbit or a chaotic orbit. The basin of attraction in Fig. 16(b) shows that there are high periodic attractors coexisting with a chaotic attractor when $\mu>-l$. It is found that in much of the parameter range, the conditions for the occurrence of period- 2 or period3 are satisfied. The only difference between the cases when $\tau_{L}$ is positive $\left(0<\tau_{L}<2 \sqrt{\delta_{L}}\right)$ and when it is negative $\left(-2 \sqrt{\delta_{L}}<\tau_{L}<0\right)$ (which basically implies a change in the sense of rotation of the spiral attractor $L^{*}$ ) is that in the negative case, a period-2 orbit is found to satisfy the conditions for stability and existence for the entire range of $\mu$. 
The Case of Positive Discontinuity: For $\mu<-l, L^{*}$ is a spiral attractor while $\bar{R}^{*}$ is a virtual flip saddle; for $\mu \in(-l, 0), L^{*}$ is a spiral attractor and $R^{*}$ is a flip saddle; for $\mu>0, \bar{L}^{*}$ is a virtual attractor while $R^{*}$ is a flip saddle. The first two cases will cause a stable period-1 orbit to exist. For the last case, the virtual attractor $\bar{L}^{*}$ maps all initial conditions in $L$ to $R$, which subsequently flip to $L$. This may either lead to a stable high-periodic orbit or a chaotic orbit.

\subsection{Flip Attractor to Flip Saddle \\ $\left(-\left(1+\delta_{L}\right)<\tau_{L}<-2 \sqrt{\delta_{L}}, \quad \tau_{R}<-\left(1+\delta_{R}\right)\right)$}

The Case of Negative Discontinuity: For $\mu<0, L^{*}$ is a flip attractor while $\bar{R}^{*}$ is a virtual flip saddle; for $\mu \in(0,-l), \bar{L}^{*}$ is a virtual flip attractor and $\bar{R}^{*}$ is a virtual flip saddle; for $\mu>-l, \bar{L}^{*}$ is a virtual flip attractor and $R^{*}$ is a flip saddle. For the first case a stable period-1 orbit exists. For the other two cases, all initial conditions in $L$ flip to the other side of $\bar{L}^{*}$ and land in $R$. Points in $R$ diverge to infinity along the unstable manifold $U_{R}$ of the flip saddle (no matter whether it is real or virtual), so that no attractor exists in the phase space.

The Case of Positive Discontinuity: For $\mu<-l, L^{*}$ is a flip attractor and $\bar{R}^{*}$ is a virtual flip saddle. A stable period- 1 orbit exists but higher periodic orbit may coexist along with the period-1 orbit. For $\mu \in(-l, 0), L^{*}$ is a flip attractor and $R^{*}$ is a flip saddle, and a period- 1 orbit exists and almost the entire space is stable. For $\mu>0, \bar{L}^{*}$ is a virtual flip attractor while $R^{*}$ is a flip saddle, and no attractor exists. There is no basin of attraction because of the diverge-to-infinity action of the unstable manifold of the flip saddle $R^{*}$ similar to the cases discussed earlier (since the attractor is virtual).

The bifurcation behavior for the case $\tau_{L}<-\left(1+\delta_{L}\right)$ and $-\left(1+\delta_{R}\right)<\tau_{R}<$ $-2 \sqrt{\delta_{R}}$ is symmetric upon varying the parameter $\mu$ in the opposite direction.

\subsection{Flip Saddle to Flip Saddle (flipping along both directions) $\left(\tau_{L}<-\left(1+\delta_{L}\right), \quad \tau_{R}<-\left(1+\delta_{L R}\right)\right)$}

No attractor exists in this case for any parameter value for both negative and positive discontinuity because of the action of the unstable manifolds of both the saddles - real or virtual. 


\section{Conclusion}

This paper contains the first attempt to analyze the bifurcation phenomena in two-dimensional discontinuous maps in terms of asymptotically stable behavior occurring for various parameter combinations. In this investigation we have used a piecewise affine approximation of the map in the neighborhood of the border, and then have partitioned the parameter space into regions depending on the types of the fixed points at the two sides of the border. For each case we have described the "typical" bifurcation phenomena in terms of the stable orbits. Where applicable, we have explained the occurrence and stability of chaotic orbits in terms of the structure of the stable and unstable manifolds of the fixed points. We do not claim to have done a complete study. Indeed, the dynamics of this system is so rich that it may take years to investigate all the possible situations.

A few atypical features of the bifurcations in discontinuous maps have been found in this study:

(1) The bifurcation behavior depends on the sign of the discontinuity;

(2) There can be stable orbits even when no fixed point exists;

(3) Chaos can occur even when each subsystem is contractive in nature;

(4) Period-incrementing sequences are common. Each transition is "hard" in the sense that the state discontinuously jumps from one periodic orbit to another.

(5) While in continuous maps, only stable manifolds of saddle fixed points can form a basin boundary, in discontinuous maps the line of discontinuity and its pre-images can also form a basin boundary.

We have used the theoretical framework developed in this paper to explain the non-standard bifurcation behavior of the Static VAR Compensator. We have shown that the system yields a discontinuous map. As the firing angle is continuously varied, there comes a parameter range where no fixed point exists. In that situation, the state jumps discontinuously to high periodic orbits that occur in this range of the parameter, as per the prediction of our theory.

We believe that this body of knowledge will help in understanding the nonstandard bifurcations observed in a number of physical systems that yield discontinuous maps on discrete-time modeling. We also hope that this work will provoke further investigation on the dynamics of discontinuous maps. 


\section{Acknowledgements}

One of the authors B. Rakshit is thankful to Council of Scientific and Industrial Research, Government of India, for a junior research fellowship.

\section{References}

[1] H. E. Nusse, J. A. Yorke, Border-collision bifurcations including "period two to period three" for piecewise smooth maps, Physica D 57 (1992) 39-57.

[2] H. E. Nusse, J. A. Yorke, Border-collision bifurcations for piecewise smooth one dimensional maps, International Journal of Bifurcation and Chaos 5 (1) (1995) 189-207.

[3] M. di Bernardo, C. J. Budd, A. R. Champneys, P. Kowalczyk, Piecewisesmooth Dynamical Systems: Theory and Applications, Springer Verlag (Applied Mathematical Sciences), New York, 2008.

[4] Z. T. Zhusubaliyev, E. Mosekilde, Bifurcations and Chaos in PiecewiseSmooth Dynamical Systems, World Scientific, Singapur, 2003.

[5] M. di Bernardo, M. I. Feigin, S. J. Hogan, M. E. Homer, Local analysis of $C$-bifurcations in $n$-dimensional piecewise smooth dynamical systems, Chaos, Solitons \& Fractals 10 (11) (1999) 1881-1908.

[6] S. Banerjee, C. Grebogi, Border collision bifurcations in two-dimensional piecewise smooth maps, Physical Review E 59 (4) (1999) 4052-4061.

[7] S. Banerjee, P. Ranjan, C. Grebogi, Bifurcations in two-dimensional piecewise smooth maps - theory and applications in switching circuits, IEEE Transactions on Circuits and Systems-I 47 (5) (2000) 633-643.

[8] A. N. Sharkovsky, L. O. Chua, Chaos in some 1-D discontinuous maps that appear in the analysis of electrical circuits, IEEE Transactions on Circuits and Systems-I 40 (10) (1993) 722-731.

[9] P. Jain, S. Banerjee, Border collision bifurcations in one-dimensional discontinuous maps, International Journal of Bifurcation and Chaos 13 (11) (2003) 3341-3352.

[10] S. J. Hogan, L. Higham, T. C. L. Griffin, Dynamics of a piecewise linear map with a gap, Proceedings of the Royal Society-A 463 (2007) 49-65.

[11] S. Banerjee, S. Parui, A. Gupta, Dynamical effects of missed switching in current-mode controlled dc-dc converters, IEEE Transactions on Circuits \& Systems - II 51 (12) (2004) 649-654.

[12] C. J. Budd, P. T. Piiroinen, Corner bifurcations in nonsmoothly forced impact oscillators, Physica D 220 (2) (2006) 127 - 145.

[13] R. Rajaraman, I. Dobson, S. Jalali, Nonlinear dynamics and switching time bifurcations of a thyristor controlled reactor circuit, IEEE Transactions on Circuits and Systems-I 43 (12) (1996) 1001-1006. 
[14] S. Jalali, I. Dobson, R. H. Lasseter, G. Venkataraman, Switching time bifurcations in a thyristor controlled reactor, IEEE Transactions on Circuits and Systems-I 43 (3) (1996) 209-217.

[15] A. L. Shilnikov, N. F. Rulkov, Subthreshold oscillations in a map-based neuron model, Physics Letters A 328 (2/3) (2004) 177-184.

[16] N. F. Rulkov, Modeling of spiking-bursting neural behavior using twodimensional map, Physical Review E 65 (4) (2002) 041922.

[17] I. Sushko, L. Gardini, T. Puu, Tongues of periodicity in a family of twodimensional discontinuous maps of real möbius type, Chaos, Solitons and Fractals 21 (2) (2004) 403-412.

[18] S. Banerjee, M. S. Karthik, G. H. Yuan, J. A. Yorke, Bifurcations in one-dimensional piecewise smooth maps - theory and applications in switching circuits, IEEE Transactions on Circuits and Systems-I 47 (3) (2000) 389-394.

[19] P. S. Dutta, B. Routroy, S. Banerjee, S. S. Alam, On the existence of low-period orbits in $n$-dimensional piecewise linear discontinuous maps, Nonlinear DynamicsDOI: 10.1007/s11071-007-9318-y.

[20] V. Donde, I. A. Hiskens, Shooting methods for locating grazing phenomena in hybrid systems, International Journal of Bifurcation and Chaos 16 (3) (2006) 671-692.

[21] K. T. Alligood, T. D. Sauer, J. A. Yorke, Chaos: An Introduction to Dynamical Systems, Springer-Verlag, 1996.

[22] S. Banerjee, J. A. Yorke, C. Grebogi, Robust chaos, Physical Review Letters 80 (1998) 3049-3052.

[23] A. Back, J. Guckenheimer, M. R. Myers, F. J. Wicklin, P. A. Worfolk, Dstool: Computer assisted exploration of dynamical systems, Notices of the American Mathematical Society 39 (4) (1992) 303-309.

[24] B. Krauskopf, H. M. Osinga, Investigating torus bifurcations in the forced van der pol oscillator, in: E. Doedel, L. Tuckerman (Eds.), Numerical Methods for Bifurcation Problems and Large-Scale Dynamical Systems, Vol. 119 of The IMA Volumes in Mathematics and its Applications, Springer-Verlag, 2000, pp. 199-208.

[25] J. P.England, B. Krauskopf, H. M. Osinga, Computing one-dimensional stable and unstable sets of planar maps without the inverse, SIAM Journal on Applied Dynamical Systems 3 (2) (2004) 161-190. 